\title{
Effect of inspiratory muscle training with an intermediate load on inspiratory power output in COPD
}

\author{
C. Villafranca, G. Borzone, A. Leiva, C. Lisboa
}

Effect of inspiratory muscle training with an intermediate load on inspiratory power output in COPD. C. Villafranca, G. Borzone, A. Leiva, C. Lisboa. CERS Journals Ltd 1998.

ABSTRACT: There is very little information about the effect of inspiratory muscle training on inspiratory flow $\left(V^{\prime} I\right)$ and thus on power output $(\mathrm{PO})$ in patients with chronic obstructive pulmonary disease (COPD). In this study we aimed to evaluate the changes induced by training on the determinants of PO.

Thirty one patients with severe COPD were randomly divided into: Group 1, trained with $30 \%$ maximal inspiratory pressure $(P I, \max )$; Group 2 , with $10 \%$ PI,max; and Group 3 also trained with $30 \% P_{I}$,max, but the breathing pattern was evaluated while performing the training manoeuvres along inspiratory muscle training (IMT). All groups used a threshold device for 10 weeks. The PO for each of the loads during an incremental threshold test was evaluated prior to and after training.

Maximal PO (POmax) increased in all groups, but the increment was higher in groups trained with $30 \% P_{I}, \max (\mathrm{p}<0.005)$, mainly due to an increase in $V^{\prime}$ I. Group 3 showed a progressive increase in $V^{\prime} I(p<0.001)$ during the training manoeuvres in spite of an increase in load along IMT. In addition, the load after IMT was overcome with a shorter inspiratory time $(t \mathrm{I})(\mathbf{p}<\mathbf{0 . 0 2})$, a smaller $t \mathrm{t} / \mathrm{total}$ duration of the respiratory cycle $(t$ tot $),(p<0.001)$ with no change in tidal volume or $t$ tot. The increment in POmax in this group correlated with the $V^{\prime} I$ generated while training $(r=0.85$; p<0.0001).

We conclude that in patients with chronic obstructive pulmonary disease, the use of an intermediate threshold load for training improves power output mainly by increasing inspiratory flow, an effect consistent with an increase in shortening velocity of inspiratory muscles.

Eur Respir J 1998; 11: 28-33.
Departamento de Enfermedades Respiratorias, Pontificia Universidad Católica de Chile, Santiago, Chile.

Correspondence: C. Lisboa

Departamento de Enfermedades Respiratorias

Pontificia Universidad Católica de Chile Marcoleta $345,4^{\circ}$ piso

Santiago

Chile

Fax: 005626335255

Keywords: Chronic obstructive pulmonary disease

inspiratory muscle training

power output

respiratory muscles

Received: August 191997

Accepted after revision November 171997

This study was funded by Fondecyt 195/ 1149 .
The effect of inspiratory muscle training (IMT) on inspiratory muscle performance has been generally evaluated through the changes it imposes on strength and/or endurance of these muscles, which mainly depend on the type of load employed for training [1]. Based on data obtained with limb skeletal muscle training, it is generally agreed that skeletal muscle training is stimuli specific [2]. Regarding respiratory muscles, TzELEPIS et al. [3] have studied the specificity of IMT in terms of changes in pressure (strength) and flow (velocity of shortening) in normal subjects. Using three different training protocols: 1) high inspiratory pressure-low flow; 2) low pressure-high flow; 3 ) intermediate inspiratory pressure and flow, they concluded that training with an intermediate load increases both maximal pressure and maximal flow in a fashion similar to that obtained using either high pressure or high flow, respectively, enhancing therefore the maximal power of inspiratory muscles. Since the endurance of respiratory muscles is related to power, these authors suggested that IMT protocols that result in an increase in power output (PO) are likely to be the most beneficial in terms of improving exercise tolerance.

We have recently demonstrated that in patients with chronic obstructive pulmonary disease (COPD), IMT using an intermediate threshold load (30\% of maximal inspiratory pressure $(P \mathrm{I}, \max ))$ increases the maximal inspiratory muscle power output (POmax) [4]. Our hypothesis is that this increase in POmax depends on the breathing pattern used for training at a particular load, and hence on the level of flow and pressure developed during the training manoeuvres. To further investigate the effects of IMT on POmax of inspiratory muscles, we studied the response to an incremental threshold loading test before and after IMT, and the relationship between the increase in POmax after IMT with: 1) the breathing pattern; and 2) the change in both pressure and flow developed during the training manoeuvres.

\section{Materials and methods}

\section{Subjects}

The study was performed on three different groups of patients who participated in two different protocols.

Protocol 1. Twenty patients with COPD were randomly allocated to IMT with either $30 \%$ (Group 1) or $10 \%$ (Group 2) of peak PI,max as a training load. Their baseline characteristics are summarized in table 1 . In these two groups of patients we assessed the effects of IMT on 
Table 1. - Baseline characteristics of the patients

\begin{tabular}{lccc}
\hline & Group 1 & Group 2 & Group 3 \\
& $30 \%$ PI,max & $10 \%$ PI,max & $30 \%$ PI,max \\
\hline Patients n & 10 & 10 & 11 \\
Sex M/F & $6 / 4$ & $7 / 3$ & $6 / 5$ \\
Age yrs & $61 \pm 1.7$ & $64 \pm 1.6$ & $71 \pm 3.0$ \\
FEV 1/FVC \% & $40 \pm 3$ & $38 \pm 1.8$ & $42 \pm 2.4$ \\
$P_{\mathrm{I}, \max \mathrm{cmH}_{2} \mathrm{O}}$ & $70 \pm 5$ & $68 \pm 4.5$ & $61 \pm 4.2$ \\
POmax & $24 \pm 6$ & $27 \pm 2.7$ & $21 \pm 4.0$ \\
$\mathrm{cmH}_{2} \mathrm{O} \cdot \mathrm{L} \cdot \mathrm{s}^{-1}$ & & & \\
\hline
\end{tabular}

Values are absolute values or mean \pm SEM. $P$ I,max: maximal inspiratory pressure; M: male; F: female; $\mathrm{FEV}_{1}$ : forced expiratory volume in one second; FVC: forced vital capacity; POmax: maximal power output.

pressure and inspiratory flow ( $\left.V^{\prime} \mathrm{I}\right)$ during breathing against externally progressive added threshold loads, while measuring the POmax.

Protocol 2. Group 3 included 11 other COPD patients who were trained with $30 \%$ PI,max. In addition to the measurement of POmax, in these patients, we evaluated the changes in $V^{\prime}$ I and in the pattern of breathing adopted during the training manoeuvres with the threshold inspiratory trainer, along the training period. Their baseline characteristics are summarized in table 1.

The inclusion criteria were: chronic nonreversible airway obstruction with a forced expiratory volume in one second (FEV1)/forced vital capacity (FVC) less than $60 \%$, stability of their disease; and presence of dyspnoea during daily life activities. Patients with cardiac, neoplastic or other diseases that could interfere with the training protocol were excluded.

\section{Methods}

During the month prior to entering the training programme, baseline measurements were performed on patients of both protocols. Peak PI,max was measured once a week and PO on two occasions. After this period, the patients in Groups 1 and 2 were assigned by a random and double blind fashion, for training with either $30 \%$ or $10 \%$ of peak PI,max, according to LARSON et al. [5] and to our previous experience [4]. The last level corresponds to the minimal load of the training device. All patients in protocol 2 were trained with $30 \% P \mathrm{I}$,max.

Peak $P \mathrm{I}$,max was measured at functional residual capacity (FRC) during maximal inspiratory efforts against a semi-occluded airway [6], using a Validyne PM45 \pm 200 $\mathrm{cmH}_{2} \mathrm{O}$ pressure transducer (Northridge, CA, USA). To avoid the use of the buccinator muscles during the manoeuvre, the inspiratory limb of the mouthpiece had a small orifice of $1.5 \mathrm{~mm}$ acting as a leak. Pressures were recorded in a strip chart recorder (Hewlett Packard, Palo Alto, CA, USA), and the highest value of five technically satisfactory manoeuvres was used for results.

PO was measured while breathing against threshold loads using the 2 min incremental load method proposed by MARTYN et al. [7] using a modified threshold device [8]. Briefly, in a seated position, the patients breathed through the mouthpiece, with nose occluded, against the basal intrinsic load of the device and, subsequently, external weights were added every $2 \mathrm{~min}$. The initial weight was
$12.5 \mathrm{~g}$ followed by $25 \mathrm{~g}$ increments. In order to open the inspiratory valve and thus allow an inspiratory flow, patients had to generate a progressively negative threshold pressure $(P$ th $)$ to overcome the increasing weight. The test ended when the patient was unable to generate flow or had to stop because of dyspnoea assessed by the Borg's scale [9]. The inspiratory mouth pressure and flow were simultaneously recorded, and the mean values obtained during the last $30 \mathrm{~s}$ of breathing with each load were used for results. Values of $P$ th and $V^{\prime}$ I for each incremental load were used to assess the performance of each patient during the test. The inspiratory muscle PO was calculated by multiplying peak $V^{\prime}$ I by peak $P$ th for each load.

In the second protocol, the breathing pattern, $V^{\prime} I$ and mouth pressure were recorded while the patients were performing the training manoeuvres. These measurements were done on a weekly basis prior to and during the training period. For the analysis of results, the mean values obtained during the baseline period were compared with those obtained after 5 and 10 weeks of training.

Tidal volume $(V \mathrm{~T})$, inspiratory time $(t \mathrm{I})$ and total duration of the respiratory cycle ( $t$ tot) were measured, whereas mean inspiratory flow $(V \mathrm{~T} / t \mathrm{I})$ and $t \mathrm{I} / t$ tot were calculated.

Training was carried out at home using a threshold inspiratory trainer (Healthscan Products Inc., NJ, USA) for 15 min twice a day and for 6 days a week. Each week, patients returned to the laboratory to have their PI,max measured in order to adjust the load, and in the case of the patients in the second protocol, to record their breathing pattern during the training manoeuvres.

Informed consent was obtained from all patients. The protocol was approved by our institutional ethics review board.

The results were analysed using the Student's t-test for paired and unpaired samples, multiple linear regression analysis and analysis of variance (ANOVA) for repeated measures. A p-value of less than 0.05 was considered statistically significant. Values are expressed as mean \pm SEM.

\section{Results}

\section{Protocol 1}

After a 10 week period of IMT, the PI,max increased significantly $(\mathrm{p}<0.05)$ in both groups of patients: $36 \pm 9 \%$

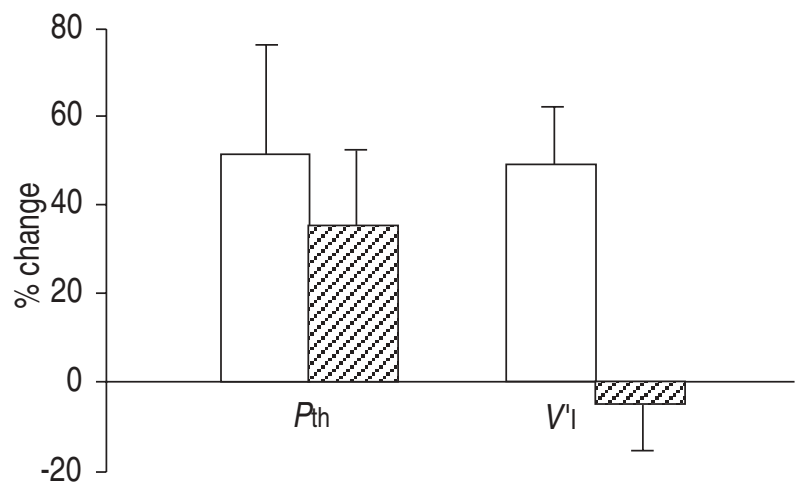

Fig. 1. - Effect of inspiratory muscle training on the two components of maximal inspiratory muscle power output. Threshold pressure (Pth) increases significantly from baseline in both groups $(\mathrm{p}<0.05)$ with training, with no significant difference between groups: inspiratory flow ( $\left.V^{\prime} \mathrm{I}\right)$ increased only in Group $1(\mathrm{p}<0.01)$. Differences between groups are statistically significant $(\mathrm{p}<0.025) . \square$ : Group $1 ; \square$ : Group 2 . 
in Group 1 and $21 \pm 5 \%$ in Group 2. Differences between groups at the end of the training period were not significant.

POmax increased in both groups: $96 \pm 14 \%$ in Group 1 $(\mathrm{p}<0.005)$ and $27 \pm 9 \%$ in Group $2(\mathrm{p}<0.025)$. The difference between groups was significant $(p<0.05)$. The effect of IMT with 10 and $30 \%$ PI,max on the two components of POmax $\left(P\right.$ th and $\left.V^{\prime} \mathrm{I}\right)$ are depicted in figure 1 as per cent change with respect to baseline data. $P$ th increased significantly in both groups $(\mathrm{p}<0.05)$, without any significant difference between groups. On the contrary, $V$ 'I showed a significant increment only in Group $1(\mathrm{p}<0.01)$, with a significant difference between groups $(\mathrm{p}<0.025)$.

Figure 2 shows several different patterns of change in inspiratory muscle PO during the incremental loading test with IMT. PO is presented in absolute values on the y-axis and the increasing loads on the $\mathrm{x}$-axis. Loads are shown as $P$ th in $\mathrm{cmH}_{2} \mathrm{O}$. Patients are grouped according to the response pattern. An increase along both axes means that the increase in PO after IMT was due to an increase in both $P$ th and $V^{\prime}$ I. An increase in PO with no increment in $P$ th indicates that only $V^{\prime}$ I increased with training. In six patients of Group 1, the increment of PO was due to an increase in both $P$ th and $V^{\prime}$ I. None of the patients in Group 2 exhibited this pattern (fig. 2a). Two patients of each group increased $V^{\prime}$ I without any change in $P$ th (fig. $2 b$ ). Four patients in Group 2 and none in Group 1 increased only $P$ th (fig. 2c). No changes were observed in two
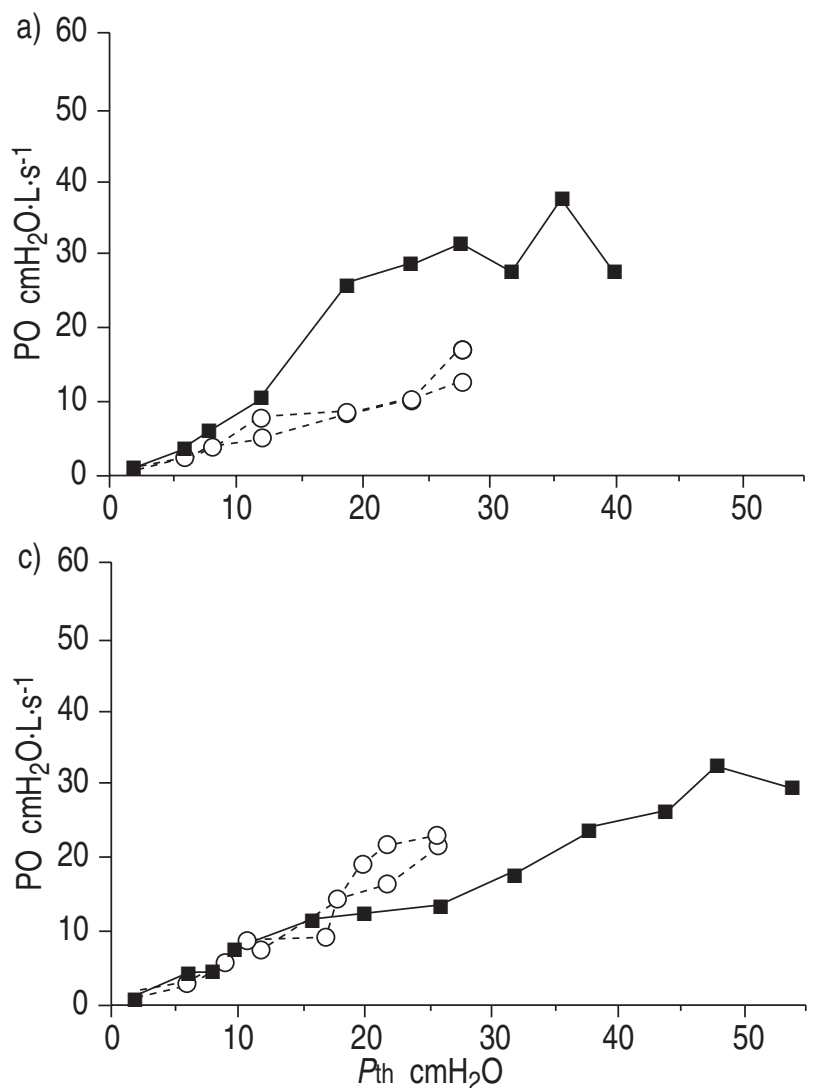

patients of Group 1 and in four patients of Group 2 (fig. 2d).

Figure 3 shows the effect of 10 weeks of IMT on PO for each of the loads during an incremental threshold loading test in both groups of patients. Data are expressed as per

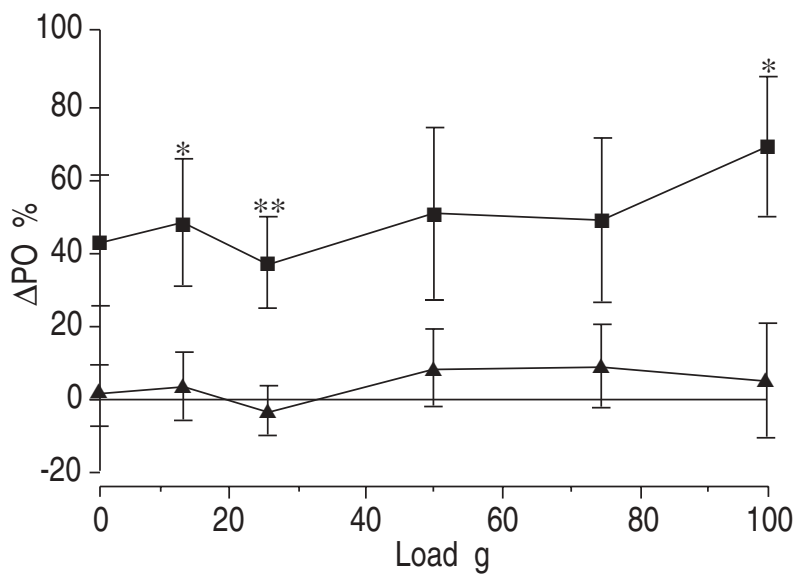

Fig. 3. - Per cent change in power output (ýPO) (mean \pm SEM) during the incremental loading test after 10 weeks of inspiratory muscle training for patients in Group 1 (घ) and in Group 2 (A). Group data for the incremental test are shown only up to a load that was overcome by all subjects during the baseline test. Patients in Group 1 showed approximately $40 \%$ increase in power output (PO) with all loads. Subjects in Group 2 did not show a significant increase in PO with these loads. *: $\mathrm{p}<0.05$; **: $\mathrm{p}<0.01$, compared to Group 2.
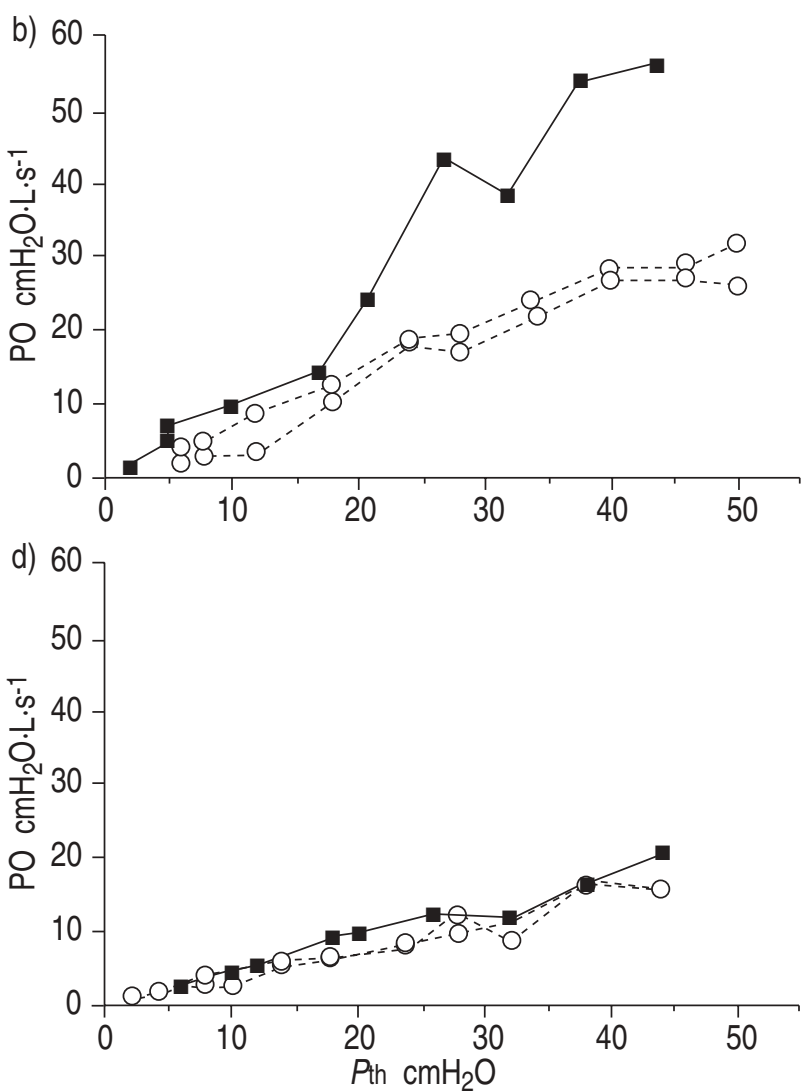

Fig. 2. - Different patterns of change in inspiratory muscle power output (PO) during the incremental loading test. Each graph illustrates complete incremental tests prior to and after 10 weeks of inspiratory muscle training (IMT). Two baseline tests are shown (- - - -) together with the test performed after 10 weeks of IMT (- - ) a) The increment in PO was due to an increase in both inspiratory flow ( $\left.V^{\prime} \mathrm{I}\right)$ and threshold pressure $\left(P_{\text {th }}\right)$. This pattern was observed in six patients in Group 1 and in none from Group 2. b) Increase in PO due to a larger $V^{\prime}$ I was seen in two subjects from each group. c) Four patients from Group 2 and none in Group 1 exhibited an increase in PO due to an increase in $P$ th only. d) Two patients in Group 1 and four in Group 2 showed no change in PO with training. 
cent of change in PO at each load during the test, only up to $100 \mathrm{~g}$, which was the load that all patients could attain in the baseline test prior to training. In Group 1, PO increased $\sim 40 \%$ at all loads, no changes were observed for patients in Group 2. Significant differences between groups were observed at some of the loads.

\section{Protocol 2: Group 3.}

In this group of patients, $P I$, max increased $31 \pm 4.2 \%$ $(\mathrm{p}<0.005)$ and POmax 78 $\pm 24 \%(\mathrm{p}<0.005)$.

Breathing pattern during the training manoeuvres. To evaluate the strategy used by patients to cope with the training loads, we plotted the pressure developed at the mouth $\left(P\right.$ th) during the training manoeuvres against the $V^{\prime}$ I before and after training. Figure 4 shows the mean values of $P$ th and $V^{\prime}$ I before and after 5 and 10 weeks of training. A progressive increase in $V^{\prime} \mathrm{I}(\mathrm{p}=0.001)$ and $P$ th $(\mathrm{p}<0.001)$ is observed. The respiratory cycle during training was characterized by a progressive shortening of $t \mathrm{I}(\mathrm{p}<0.02)$ and $t \mathrm{I} /$ tot $(\mathrm{p}<0.001)$, and a tendency to increase of $V \mathrm{~T} / \mathrm{tI}(\mathrm{p}<0.077)$ without any changes in $V$ T (fig. 5). Figure 6 shows the

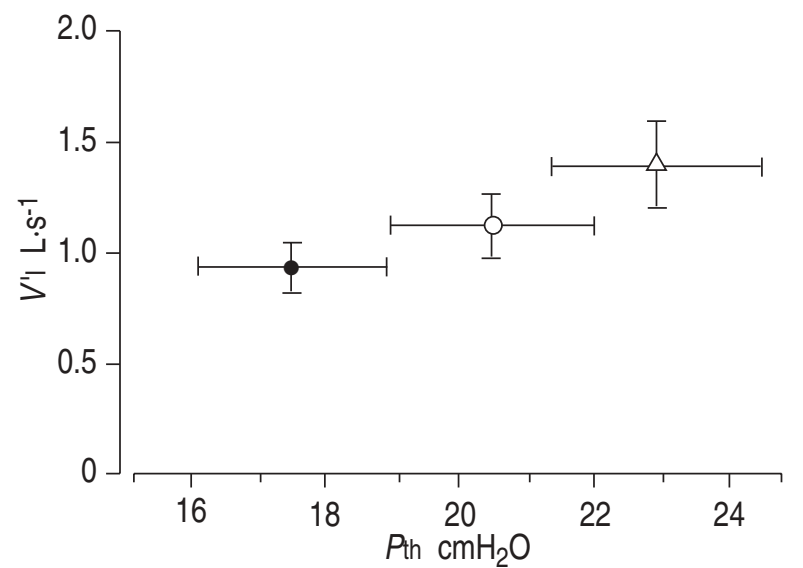

Fig. 4. - Relationship between changes in threshold pressure at the mouth level $\left(P_{\mathrm{th}}\right)$ and inspiratory flow $\left(V^{\prime} \mathrm{I}\right)$, while performing the training manoeuvres before $(\bullet)$ and after $5(\bigcirc)$ and 10 weeks (ý) of inspiratory muscle training for patients in Group 3. Results are presented as mean \pm SEM. Significant increments in both indexes were observed $(p<0.001$ and $\mathrm{p}=0.001$, respectively, analysis of variance (for repeated measures).

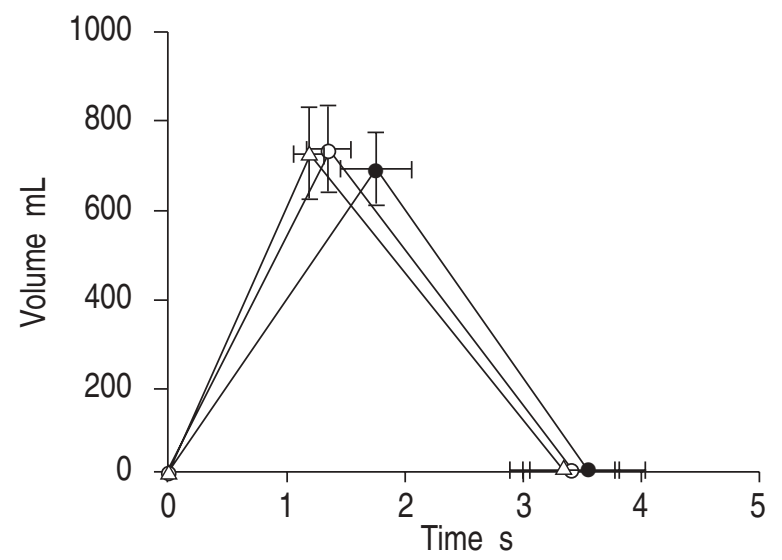

Fig. 5. - Respiratory cycle during the training manoeuvres before (๑) and at $5(\mathrm{O})$ and 10 weeks (ý) of inspiratory muscle training in patients from Group 3. Significant changes in inspiratory time $(t \mathrm{I})$ and in $t \mathrm{I} / \mathrm{total}$ duration of the respiratory cycle ( $t$ tot) are seen. Results are presented as mean \pm SEM. For further details, refer to text.

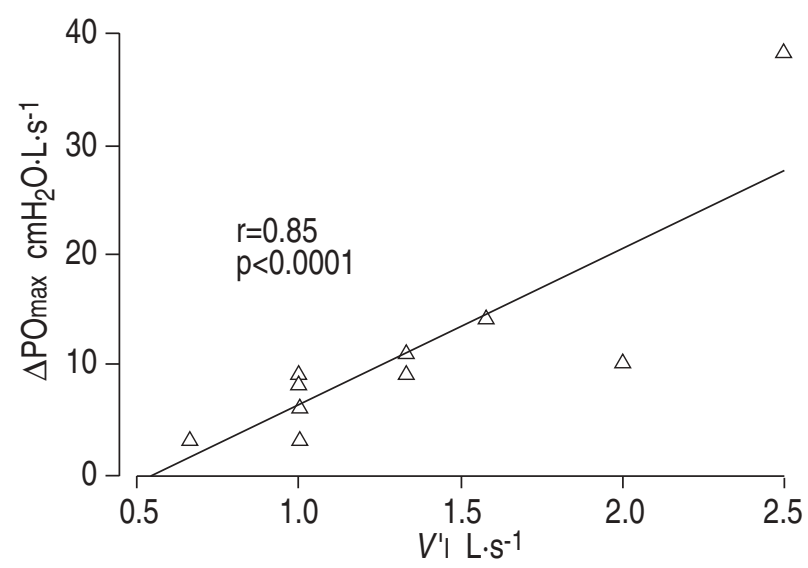

Fig. 6. - Relationship between the changes in maximal power output (ýPOmax) induced by inspiratory muscle training and the inspiratory flow $\left(V^{\prime} \mathrm{I}\right)$ generated during the training manoeuvres.

relationship between the changes in POmax induced by IMT and the $V^{\prime} \mathrm{I}$ generated during the training manoeuvres $(\mathrm{r}=$ $0.85 ; \mathrm{p}<0.0001)$.

\section{Discussion}

The results of the present study indicate that in patients with COPD, IMT using a threshold load of $30 \%$ PI,max increases inspiratory muscle PO. This change in PO was mainly due to an increase in $V^{\prime}$ I and correlated with the magnitude of the $V^{\prime}$ I developed while performing the training manoeuvres. Although POmax also improved in patients trained with a lower load $(10 \%$ PI,max), the effect in this group was smaller and mainly due to an increase in pressure with no change in flow. In addition, after IMT, the breathing pattern developed during the training manoeuvres changed to one characterized by a shorter $t \mathrm{I}$, a decreased $t \mathrm{I} / t$ tot ratio, and no significant change in $V \mathrm{~T}$ or $t$ tot.

This study corresponds to another analysis [10], which investigated the clinical effects of IMT on exercise performance in a group of patients with COPD. In addition to the increase in $P I$,max, we found in that study a reduction in dyspnoea and an increase in the 6 min walking distance.

We believe that our findings are likely to be due to a true effect of IMT on PO of the inspiratory muscles and not only due to a learning effect or to changes in the strategy to cope with the load, since in the group trained with a higher load, there are important clinical effects associated to the increase in PO. In the group trained with a lower load, the increment in POmax was mild and mainly due to an increase in $P$ th. An increment in PO due to an increase in flow was only seen in two patients in this group.

FAULKNER [2] has stated that training elicits specific structural and functional changes in skeletal muscle which depend on the stimulus used for training. Thus, training with high force contractions increases maximal force, whereas training with high velocity, low force contractions, increases maximal shortening velocity.

With regard to the effects of training on respiratory muscles, these are also shown to be stimulus specific. Recent data however, suggest that by using intermediate loads it is possible to obtain responses that involve concomitant training for both strength and endurance [5]. 
As has been pointed out by several authors [11-13], the best system for training the inspiratory muscles has yet to be defined. Results of different training protocols depend on several factors such as the magnitude and modulation of the load, the shortening velocity and the breathing pattern. In fact, the use of different modalities of ventilatory training may explain the still-controversial results obtained, especially in COPD patients [13].

With regard to the type of training protocol, BeLman et al. [14] studied the workload imposed by different training devices and breathing strategies upon the ventilatory muscles of patients with COPD. They selected six different methods going from high flow and low pressure to low flow and high pressure tasks, resulting in very different ventilatory muscle loading. A targeted resistance or a threshold device with a load of $30 \% P$ I,max, together with a respiratory frequency of 30 breaths. $\mathrm{min}^{-1}$ were found to combine a load for both pressure and flow. These types of load appear to be the best for improving PO. Theoretically, a training protocol that improves $\mathrm{PO}$, as found in our COPD patients, should be more useful than training only for strength, since the endurance of the inspiratory muscles is related to their PO [3].

There is very little information about the effect of IMT on inspiratory muscle PO in both normals and patients with COPD. Using an intermediate resistive load, TzELEPIS et al. [3] trained normal subjects and found an increment in both $P \mathrm{I}$,max and $V^{\prime} \mathrm{I}$, which is indicative of an improvement in PO. In a previous study we reported an increment in POmax after 5 weeks of IMT with an intermediate inspiratory threshold load in patients with COPD [4]. In the present study, we have also evaluated PO for each of the loads during an incremental threshold loading test, prior to and after IMT with both $10 \%$ and $30 \%$ PI,max. Figure 3 shows that after training with $30 \%$ PI,max, each load in the incremental threshold test is overcome with a flow that is approximately $40 \%$ higher than in the baseline incremental test. This increase in $V^{\prime} \mathrm{I}$ and, therefore, in PO, is probably more relevant for the patients than the increment in PI,max to cope with the ventilatory demands of daily life activities. No significant changes in PO during the incremental test were seen after training with $10 \%$ $P$ I,max.

Another study by BELMAN and SHADMEHR [15] has assessed the effects of targeted resistive breathing training, using the highest endurable load. Although they did not measure the effect on PO, they speculated that the significant increase in mean and peak mouth pressure as well as in the work of breathing during loaded breathing, were indicative of an improvement in PO.

The effect of IMT with either flow or pressure as a target load has recently been studied by TzELEPIS et al. [16] in normal subjects. They found that flow-targeted training diminished the time to reach the peak oesophageal pressure by about $25 \%$, when performing maximal inspiratory efforts. On the contrary, no such change was observed with pressure training. According to these authors, this finding is consistent with an increase in the velocity of shortening of the inspiratory muscles due to training. Further support for an increase in shortening velocity of respiratory muscles has been given in animals by the study of Bisschop et al. [17]. After 8 weeks of IMT using a moderate resistive load in rats, they found a reduction in the time to reach the peak tension ( $t \mathrm{PT})$ in an in vitro diaphragm prep- aration. These investigators attributed this physiological effect of training to specific structural changes in the diaphragm, namely hypertrophy of type IIa and IIx/b fibres [17].

We have evaluated the effect of IMT on the velocity of shortening of the inspiratory muscles in our patients in a rather indirect way, by analysing the changes in $t \mathrm{I}, t \mathrm{I} / t$ tot, $V^{\prime} \mathrm{I}$ and $V \mathrm{~T} / t \mathrm{I}$ during the training manoeuvres. The changes observed are also consistent with the notion that there is an increase in shortening velocity of the inspiratory muscles. In a previous study we showed a similar effect on the duty cycle during incremental threshold loading [4]. This pattern of breathing could be useful during exercise since with this breathing strategy, the patients could be capable of maintaining $V \mathrm{~T}$ in spite of decreasing $t \mathrm{I}$. FLYNN et al. [18] evaluated this effect of IMT on the pattern of breathing during exercise and failed to show changes in duty cycle during maximal exercise, even though during resting and threshold breathing, they did find a shorter $t \mathrm{I}$ and $t \mathrm{I} / t$ tot ratio after training.

In our study we used an intermediate threshold load with a mean respiratory frequency of 17 breaths $\cdot \mathrm{min}^{-1}$ chosen spontaneously by the patients and which was not modified by training. The main finding was the increase in PO due to a larger $V^{\prime} \mathrm{I}$ as well as to a reduction in $t \mathrm{I}$ and $t \mathrm{I} / t$ tot with a normal $V \mathrm{~T}$. These results, which are in agreement with data from Tzelepis et al. [16] and Bisschop et al. [17], suggesting changes in shortening velocity of in-spiratory muscles, allow us to speculate upon structural changes at the level of muscle fibres in response to specific recruitment patterns induced by training.

Since myosin heavy-chain composition is one of the determinants of shortening velocity [19], a possible mechanism underlying the improvement in PO with training may be the changes in myosin heavy-chain subtypes shown in the diaphragm of experimental animals [19]. GEA et al. [20] have recently shown that in dogs, resistive breathing for $2 \mathrm{~h}$ during four consecutive days, increased the messenger ribonucleic acid (mRNA) expression of specific myosin heavy-chain isoforms in the diaphragm. Further research is needed, however, to establish the role of such changes in the increase in PO due to inspiratory muscle training.

In conclusion, the results of the present study, as well as those reported by others in normal subjects $[3,16]$ suggest that the use of moderate loads to train inspiratory muscles elicits improvements in both strength and shortening velocity and thus, in power output. We believe that in patients with chronic obstructive pulmonary disease, these outcomes are more relevant for coping with the increased ventilatory demands of daily life activities, than the improvement in strength alone achieved by training with high pressure loads [1].

\section{References}

1. Leith DH, Bradley M. Ventilatory muscle strength and endurance training. J Appl Physiol 1976; 41: 508-516.

2. Faulkner JA. Structural and functional adaptations of skeletal muscle. In: Roussos C, ed. The Thorax. New York, Dekker, 1996; pp. 269-294.

3. Tzelepis GE, Vega DL, Cohen ME, Fulambarker AM, Patel KK, McCool FD. Pressure-specificity of inspiratory muscle training. J Appl Physiol 1994; 77: 795-801. 
4. Lisboa C, Muñoz V, Beroíza T, Leiva A, Cruz E. Inspiratory muscle training in chronic airflow limitation: comparison of two different training loads using a threshold device. Eur Respir J 1994; 7: 1266-1274.

5. Larson JL, Kim MJ, Sharp JT, Larson DA. Inspiratory muscle training with a pressure threshold breathing device in patients with chronic obstructive pulmonary disease. Am Rev Respir Dis 1988; 138: 689-696.

6. Black LF, Hyatt RE. Maximal respiratory pressures. Normal values and relationship to age and sex. Am Rev Respir Dis 1969; 99: 696-702.

7. Martyn JB, Moreno RH, Paré PD, Pardy RL. Measurement of inspiratory muscle performance with incremental threshold loading. Am Rev Respir Dis 1987; 135: 919923.

8. Farbinger F, Yunge M, Díaz O, Moreno R, Villarroel L, Lisboa C. Potencia máxima de los músculos inspiratorios. Enferm Resp Cir Tor 1989; 5: 64-68.

9. Borg GAV. Psychophysical bases of perceived exertion. Med Sci Sports Exerc 1982; 14: 377-382.

10. Lisboa C, Villafranca C, Leiva A, Cruz E, Pertuzé J, Borzone G. Inspiratory muscle training in chronic airflow limitation: effect on exercise performance. Eur Respir $J$ 1997; 10: 537-542.

11. Gosselink R, Decramer M. Inspiratory muscle training: where are we? Eur Respir J 1994; 7: 2103-2105.

12. Goldstein RS. Ventilatory muscle training. Thorax 1993; 48: 1025-1033.
13. Smith K, Cook D, Guyatt GH, Madhavan J, Oxman AD. Respiratory muscle training in chronic airflow limitation: a meta-analysis. Am Rev Respir Dis 1992; 145: 533-539.

14. Belman MJ, Botnick WC, Nathan SD, Chon KH. Ventilatory load characteristics during ventilatory muscle training. Am J Respir Crit Care Med 1994; 149: 925-929.

15. Belman MJ, Shadmehr R. Targeted resistive ventilatory muscle training in chronic obstructive pulmonary disease. J Appl Physiol 1988; 65: 2726-2735.

16. Tzelepis GE, Kadas V, Anthi A, et al. Inspiratory muscle contractile properties following pressure or flow training. Am J Respir Dis Crit Care Med 1997; 155: A920.

17. Bisschop A, Gayan-Ramirez G, Rollier H, et al. Intermittent inspiratory muscle training induces fiber hypertrophy in rat diaphragm. Am J Respir Crit Care Med 1997; 155: 1583-1589.

18. Flynn MG, Barter CE, Nosworthy JC, Pretto JJ, Rochford PD, Pierce RJ. Threshold pressure training, breathing pattern, and exercise performance in chronic airflow obstruction. Chest 1989; 95: 535-540.

19. Bottinelli R, Schiaffino S, Reggiani C. Force-velocity relations and heavy chain isoform compositions of skinned fibres from rat skeletal muscle. J Physiology 1991; 437: 655-672.

20. Gea J, Hamid Q, Comtois N, Salazkin I, Goldspink G, Grassino A. mRNA expression of slow heavy chain myosin of diaphragm increases following resistive breathing. Am J Respir Crit Care Med 1996; 153; A294. 